Pak. j. sci. ind. res. Ser. B: biol. sci. 2019 62B(3) 148-158

\title{
Sequence Analysis and Expression Study of LTP7 Promoter Isolated from Cotton (Gossypium hirsutum L.)
}

\author{
Ammara Masood $^{\mathrm{b} *}$, Hira Mubeen ${ }^{\mathrm{b}}$ and Nadia Iqbal ${ }^{\mathrm{a}}$ \\ ${ }^{a}$ National Institute for Biotechnology and Genetic Engineering (NIBGE), Faisalabad, Pakistan \\ ${ }^{b}$ University of Central Punjab (UCP), Lahore, Pakistan
}

(received March 5, 2018; revised June 4, 2018; accepted July 2, 2018)

\begin{abstract}
Lipid transfer proteins (LTPs) have role in transfer of phospholipids along biological membranes. A cotton LTP7 promoter was isolated using high throughput genomic sequences (HTGS) data base. Analysis of promoter nucleotide sequence revealed a number of crucial regulatory elements including core promoter elements. A $1.8 \mathrm{~kb}$ fragment of LTP7 promoter was isolated from genomic DNA of cotton and finally cloned in plant expression vector to characterize its functionality. Transient GUS assay revealed that promoter showed expression in cotton fibres during the time of elongation and different stages of secondary cell wall synthesis. Deletion analysis at $5^{\prime}$ end showed that $1 \mathrm{~kb}$ promoter showed strong expression during stage of secondary cell wall synthesis. Whereas, $1.5 \mathrm{~kb}$ deletion fragment exhibited less strong expression in cotton fibres. Results of this present study, showed that $1 \mathrm{~kb}$ deletion fragment and $1.8 \mathrm{~kb}$ LTP7 promoter exhibits fibre specific expression and may be used to express fiber genes in cotton.
\end{abstract}

Keywords: lipid transfer protein, sequence analysis, promoter, transient GUS expression, cotton fibre

\section{Introduction}

Fibre development is complex process involving large number of genes that express during specific fibre development phase. Promoters have key role in controlling expression of genes. Upstream regulatory sequences of many cotton fibre genes shows tissue specific or fibre specific expression. Some of LTP genes are expressed distinctly in developing cotton fibres. LTPs are conserved proteins found in epidermal tissues of higher plant. Lipid transfer proteins play role in shuttling of phospholipids and other fatty acid groups between lipid bilayer and responsible for cell wall biogenesis (Allen et al., 2009). These proteins are thought to have role in cuticle deposition and secretory pathway (McGee et al., 1994), plant defense against pathogens attack (Gomes et al., 2003) and plant development (Fleming et al., 1992). Promoters of fibre related genes exhibited strong expression in tobacco, Arabidopsis and cotton fibres (Machando et al., 2009). Many of LTP tobacco plant transformed with homologues of LTP genes promoter showed good expression in leaf, stem trichomes along with vascular tissues and leaf margins (Liu et al., 2000). In order to control fibre specific expression the study of promoter and regulatory region of fibre specific genes is very

*Author for correspondence;

E-mail: ammara.masood@ucp.edu.pk helpful. Therefore, need of the hour is to develop cotton varieties with superior quality fibre to avoid foreign exchange as well as to export it to other international market at high prices.

The present study describes isolation and sequence analysis of cotton lipid transfer protein (LTP7) promoter from cotton. Sequence analysis showed important regulatory motifs for promoter activity. Full length 1.8 $\mathrm{kb}$ promoter was expressed in cotton fibres. 5 ' Deletion mutants of $1 \mathrm{~kb}$ and $1.5 \mathrm{~kb}$ also showed GUS related activity. Although, $1 \mathrm{~kb}$ fragment showed strong expression than $1.5 \mathrm{~kb}$ fragment. In future they may be able to derive expression of GUS reported gene in cotton fibre. Therefore, LTP7 promoter and its $1 \mathrm{~kb}$ promoter may be used to express transgene in cotton fibre to improve cotton fibre quality.

\section{Materials and Methods}

An upstream region LTP7 gene from cotton was selected for promoter isolation. Nucleotide sequence of LTP7 was extracted and blast searched in HTGS (high throughput genomic sequences) at NCBI. Nucleotide sequence that were matched with HTGS of cotton was taken and its exact coding frame were identified using Expasy tool. Two different upstream regions of $2 \mathrm{~kb}$ and $1.8 \mathrm{~kb}$ were analyzed using protein blast (Blast P) and then both were patent searched in patent blast. 
Finally, about $1.8 \mathrm{~kb}$ upstream region of LTP7 gene was chosen for promoter evaluation and characterization. Their transcription initiation site was found by fruitfly database and further bioinformatics analysis was performed using Plant pan and PlantCARE.

LTP7 promoter was isolated from genomic DNA of cotton by PCR amplification using 5' TATGAGCTCGAGTGCAATATTGGCTTACC3'and 5'GACAAGCTTGACTGAAAAAATCCACCAAAC $3^{\prime}$ as forward and reverse primer. PCR product was further cloned in TA cloning vector. Next, they were cloned in expression vector pGR1. To study the expression effect and to study the regulatory motifs $1.5 \mathrm{~kb}$ and $1 \mathrm{~kb}$ promoter were also cloned in pGR1. Their transient expression was analyzed using GUS particle bombardment. Normal promoters and experimental 35S promoter and promoter less constructs were bombarded at same conditions. Cotton tissues bombarded with promoter constructs were dipped in GUS staining solution containing X-Gluc and placed under dark at $37{ }^{\circ} \mathrm{C}$ till appearance of blue colour stain. Different cotton tissues were decanted and washed till removal of chlorophyll from green tissues.

\section{Results and Discussion}

Based on expression profiling results a highly expressed genes in cotton fibres was selected for promoter isolation, lipid transfer protein (LTP7). Nucleotide sequences of cotton LTP7 promoters were retrieved from high through put genomic sequences (HTGS) in NCBI. BLAST search of LTP gene sequence in HTGS database revealed that sequence matched with HTGS of Gossypium hirsutum, clone ZMMBBb $621 \mathrm{G} 9$ (Accession no. 202820). For LTP7 promoter 1843 bp (about $1.8 \mathrm{~kb}$ ) sequence was selected.

Evaluation of promoter sequences using bioinformatics tools. Several bioinformatics tools were used to identify transcription start site and cis-acting regulatory sequences of LTP7 promoter. Transcription initiation site was identified by promoter prediction analysis tools available at fruitfly.

TiS site (CTTTTGGCTATAAAAACCCTCCTACCC TCAAGCCCTAACCACGACAATCA)

The promoter sequence of LTP was BLAST searched against patent sequences at NCBI. Results revealed no significant homology with any reported promoter
(Table 1). LTP7 promoter sequence analysis of LTP7 promoter through PlantCARE identified various number of cis-acting regulatory motifs (Table 2). In $1.8 \mathrm{~kb}$ LTP7 full length promoter, TATA box was identified at position -23. A CAAT box having consensus sequence CAATT was also found at position -131 relative to transcription initiation site. A number of light responsive motifs namely Sp1 motif, G-box (TAGGTG), Box-4 and BoxI were also present in LTP7 promoter.

Similarly to identify various transcription factors plant pan was used, several binding sites for transcription factors were found in LTP7 promoter (Table 3). There were binding sites for MYB proteins that are involved in dehydration response. Similarly two binding sites with consensus sequence CTAACCAC involved in activation of rapid transcription were found in LTP7 promoter.

Isolation of full length cotton LTP7 promoter. Chromosomal DNA of young cotton leaves was used as template for amplification of full length promoters. Several primer pairs were tested to get amplifications. Gradient PCR for optimized annealing temperature shows that LTP7 promoter was amplified using LTPGh1 (Fig. 1). 8F3 as forward primer and LTPGhR1 as reverse primer at temperature of $48{ }^{\circ} \mathrm{C}$ (Fig. 2).

Cloning of LTP7 promoter in TA cloning vector pTZ57R/T. Amplified product of LTP7 promoters was separately purified and ligated into $\mathrm{pTZ} 57 \mathrm{R} / \mathrm{T}$ vector then transformed into competent cells (E. coli). SacI and ApaI was used for restriction digestion, Two discrete

Table 1. Patent BLAST results for LTP7 promoter

\begin{tabular}{llll}
\hline \hline Description & $\begin{array}{l}\text { Query } \\
\text { coverage } \\
(\%)\end{array}$ & $\begin{array}{l}\text { Identity } \\
(\%)\end{array}$ & $\begin{array}{l}\text { Accession } \\
\text { number }\end{array}$ \\
\hline $\begin{array}{l}\text { Sequence 38 from patent US } \\
7498428>\text { GZ } \mid 935393-1 \\
\text { sequence 38 from patent }\end{array}$ & 32 & 88 & GP23723-1 \\
$\begin{array}{l}\text { US812951 } \\
\text { Sequence 39 from patent }\end{array}$ & 32 & 80 & GP235724-1 \\
$\begin{array}{l}\text { US749828>qb GZ|953941 } \\
\text { from patent US812951 }\end{array}$ & & & \\
$\begin{array}{l}\text { Sequence from patent } \\
\text { US 5792933 }\end{array}$ & 5 & 92 & AR022671-1 \\
$\begin{array}{l}\text { Sequence 17 from patent } \\
\text { US 7732678 }\end{array}$ & 5 & 85 & GX277307-1 \\
\hline \hline
\end{tabular}


Table 2. Cis-acting elements in the LTP7 promoter (plantCARE analysis)

\begin{tabular}{|c|c|c|c|}
\hline Motif name & Organism & Sequence & Putative function \\
\hline BOX-I & Pisum sativum & TTTCAAA & Light responsive element \\
\hline BOX-4 & Petroselinum crispum & ATTAAT & Light responsiveness \\
\hline ABRE & $\begin{array}{l}\text { Arabidopsis thaliana } \\
\text { Arabidopsis thaliana }\end{array}$ & $\begin{array}{l}\text { TACGTG } \\
\text { TACGTG }\end{array}$ & Abscisic acid responsiveness \\
\hline ARE & $\begin{array}{l}\text { Zea mays } \\
\text { Zea mays }\end{array}$ & $\begin{array}{l}\text { TGGTTT } \\
\text { TGGTTT }\end{array}$ & Anaerobic responsiveness \\
\hline CAAT-Box & $\begin{array}{l}\text { Glycine max } \\
\text { Glycine max } \\
\text { Brassica rapa } \\
\text { Arabidopsis thaliana } \\
\text { Brassica rapa }\end{array}$ & $\begin{array}{l}\text { CAATT } \\
\text { CAATT } \\
\text { CAAAT } \\
\text { CCAAT } \\
\text { CAAAT }\end{array}$ & Common cis-acting element and enhancer region \\
\hline G-Box & Antirrhium majus & CACGTA & Light responsiveness \\
\hline G-box & Daucus carota & TAGGTG & Light responsiveness \\
\hline MRE & Petroselinum crispum & AACCTAA & MYB binding site involved in light responsiveness \\
\hline Sp1 & Zea mays & $\mathrm{CC}(\mathrm{G} / \mathrm{A}) \mathrm{CCC}$ & Light responsiveness \\
\hline RY element & Halianthus annus & CATGCATG & Seed specific expression \\
\hline HSE & Brassica oleracea & AAAAATTTC & Heat stress responsiveness \\
\hline MBS & $\begin{array}{l}\text { Arabidopsis thaliana } \\
\text { Arabidopsis thaliana }\end{array}$ & $\begin{array}{l}\text { CAACTG } \\
\text { TAACTG }\end{array}$ & MYB binding site for drought inducibility \\
\hline TATA-box & $\begin{array}{l}\text { Lycopersicon esculentum } \\
\text { Zea mays } \\
\text { Lycopersicon esculentum }\end{array}$ & $\begin{array}{l}\text { TTTTA } \\
\text { TTTAAAAA } \\
\text { TTTTA }\end{array}$ & Core promoter element around -30 of TSS \\
\hline TC-rich repeats & Nicotiana tabacum & ATTTTCTCTA & Stress and defense responsiveness \\
\hline TCA element & Nicotiana tabbacum & CСАTCTTTT & Salicylic acid responsiveness \\
\hline 5' UTR Py-rich stretch & Lycopersicon esculentum & ТTТСТТСТСТ & Cis-acting element conferring high transcription \\
\hline
\end{tabular}

bands of $1.8 \mathrm{~kb}$ LTP promoter and $2.8 \mathrm{~kb}$ vector backbone were obtained after digestion of recombinant clones (Fig. 3).

\section{Construction of deletion fragments of LTP7} promoter. Full length promoters cloned in TA cloning vector were used as template to generate promoter deletion fragments. Confirmation of clones was carried out through restriction analysis using SacI and HindIII (Fig. 4).

Important regulatory motifs in LTP7 promoter fragments. Deletion analysis of LTP7 promoter at 5' end resulted in deletion of a number of motifs with diverse important functions (Table 4). Full length LTP7 cotton promoter consisted of 23 binding sites for ARRIAT (aataatcacgtaggttgg) whereas its 1.5 and $1 \mathrm{~kb}$ deletion fragments consisted of 18 and 11 sites, respectively.

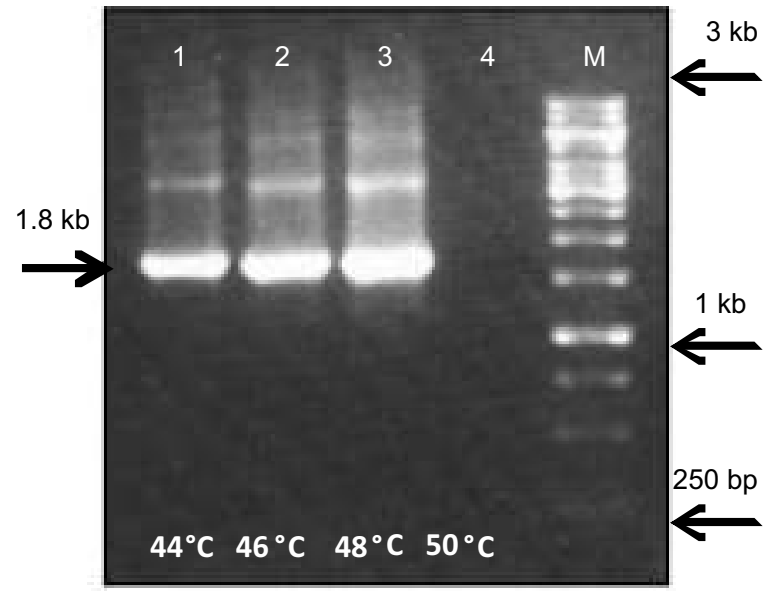

Fig. 1. Gradient PCR for amplification of LTP7 promoter. M: $1 \mathrm{~kb}$ DNA ladder; Lane 14 represent range of annealing temperatures $44,46,48$ and $50{ }^{\circ} \mathrm{C}$, respectively. 
Cloning of full promoters and their fragments into expression vector (pGR1). Finally the promoters and their deletion fragments were inserted into expression vector pGR1 by substituting the widely used $35 \mathrm{~S}$ promoter upstream of GUS gene. pGR1 vector was restricted with SacI and HindIII to excise 750 bp 35S promoter fragment. Full length LTP7 promoter and its deletion fragments were amplified through PCR and restricted with SacI and HindIII for ligation into restricted vector pGR1. Resultant clones were confirmed after restriction digestion with SacI and HindIII (Fig. 5). 1 kb LTP7 promoter was named pGRLTP1, $1.5 \mathrm{~kb}$ promoter was named pGRLTP1.5, and pGRLTP1.8 having $1.8 \mathrm{~kb}$ LTP7 promoter. Physical maps of pGRLTP1, pGRLTP1.5 and pGRLTP1.8 are given in Fig. 6A-C.
Transient GUS expression studies. The main objective of present research was to isolate novel plant promoters to obtain high transgene expression. So, to evaluate and analyze the promoter activity of isolated promoters and their deletion fragments transient GUS assay was performed. Along with full length promoter two deletion fragments of 1.5 and $1 \mathrm{~kb}$ were cloned in expression vector pGR1 and their expression was determined through transient GUS assay. Cotton fibre and various tissues were used as explants to determine experimental accuracy along with positive and negative controls. In order to monitor false results, promoter less constructs were bombarded on cotton tissues to get negative results. No GUS expression was detected in any tissues as shown in Fig. 7. pGR1 vector having 35S promoter was taken as positive control. GUS expression was detected

Table 3. Transcription factor binding sites in LTP7 promoter (plantPAN)

\begin{tabular}{|c|c|c|c|}
\hline Motif & Sequence & Function & Organism \\
\hline ABRELATERD1 & CACGT & Etiolated induced expression & Arabidopsis \\
\hline ACGTATERD1 & ACGT & Dehydration or dark responsiveness & Arabidopsis \\
\hline AGL3 & CTATTTATGG & Regulator of transcription & Arabidopsis/pea \\
\hline ANAERO1CONSENSUS & AAACAAA & Anaerobic induction & Arabidopsis \\
\hline ARRIAT & $\begin{array}{l}\text { aataatcACGT } \\
\text { aggttgg }\end{array}$ & Transcription activation & Arabidopsis \\
\hline CARGCWBGAT & TTATTATAG & Binding site for MADS protein & Arabidopsis \\
\hline AtMYB2 & CTAACCA & Drought \&ABA responsiveness & Arabidopsis \\
\hline AtMYC2 & CACATG & ABA signaling & Arabidopsis \\
\hline CСAАТВOX1 & ATTGG & Heat responsiveness & Carrot/Arabidopsis \\
\hline DPBFCOREDCDC3 & ACACATG & Embryo related motif & Soybean \\
\hline GT1GMSCAM4 & TTTTTC & Pathogen/salted induced response & Arabidopsis \\
\hline MYCATRD22 & CATGTG & Dehydration response & Tomato/Lycopersicon \\
\hline ERELEE4 & AATTCAAA & Ethylene responsiveness & Arabidopsis \\
\hline GAREAT & TTTGTTA & GA induced response & Petunia/Arabidopsis \\
\hline GATABOX & TATC & High transcription & Arabidopsis \\
\hline P1F3 & aataatCACGTaggttgg & Phytochrome signaling & Arabidopsis \\
\hline GT1CONSENSUS & GGTAAA & Light responsiveness & Tobacco \\
\hline INRNTPSADB & TTCAGTTC & Initiation element of transcription & Arabidopsis \\
\hline MYB1AT & TAACCA & Dehydration response & Arabidopsis \\
\hline SEF3MOTIFGM & TGGGTT & Light responsiveness & Arabidopsis \\
\hline MYB2AT & TAACTG & Water stress response & Arabidopsis \\
\hline REBETALGLHCB2 1 & TATCCG & Phytochrome response & Arabidopsis \\
\hline RHERPATEXPA7 & TCACGT & Root hair specific expression & Potato \\
\hline SEF1MOTIF & ATATTTAAT & Embryo specific expression & Arabidopsis \\
\hline SREATMSD & GGATAA & Sugar responsiveness & Potato \\
\hline SURE1STPAT21 & TTTTCTATT & Sucrose responsiveness & Arabidopsis \\
\hline SORLREP3AT & GTTTGGTG & Light responsiveness & Potato \\
\hline MYBST1 & GGATA & Transcription activation & Arabidopsis \\
\hline SV40COREENHAN & CTAACCAC & Enhancer element & Arabidopsis \\
\hline TBOXATGAPB & CAAAGT & Light responsiveness & Arabidopsis \\
\hline
\end{tabular}


-1752 TAGATTTACCAGTGAACAAATCTAGAGAAGAAATTAGTCGAACTACATCAATGTCGAGAAGATCGGGTAGAGATAGAAAG - 1673 -1672 ACAATCTGATTTCAGGGGTATTCGATAGACCTCCAGCAAAGTGTGAAGCAGTGTTCAGAATGTTCCCCCGGGCCTAAGTT -1593 -1592 GTCAATATTGTGGAAGATATTCATTTCAGAGAATGCAGGACCAAGATGGGGGGCTTTGTTAAAAATGGTGGGGGGTACTA -1513 -1512 TTCATCTTAATTCGAGATTGTCCCGACTTATAAAAAAGATGAATTGGGAACAGAAAAGAAATACAGAGAAAACCATTTCC - 1433 -1432 CCCAAAAAATAGACGTTCGGGGCAGAACAGTGCTTCCTGGGACTACCCCGTTCGGGGACGAAGGAATCAGTTGGGCGAT -1353 -1352 CAAAGAATAAAGCACCAGCACGTACCTCCCCCCTTTCTAGCAAAGGAAAGAGGTACCGCCTCCCGGAGGAAATTGCTGG -1273 -1272 GACCTTTTCTATCTTTTTTGATGTTAGGTTTATGCAATTGAAAAGACCCCTGGAACCACTCCCTCCCATATTTTTGCACTA -1193 -1192 TGGTTGCATCTAAAAAAAAAAATTAACTGTTTGAGTCCCCTGGAATTTTTAAGAAGTGGGGAAGATAATTAATCCTTCTT -1113 -1112 GTTTTTCAGTTATTTAGGGTTGTGTTTTGGGTTTTTTCATTTTATAAATGTTGAGAGCTAAATTTATTGGATTATTTAGAA -1033 -1032 TTTGAACTAAAATGAAAAATATTGTAAACATTGAGGAATAATTTTGTTGAATTTTAATTTTTATATTTAAGGCCAAATTAA -953 -952 TAAAATATAGTAAACATTAGATAACTAAATTTATTATTAGAATCCACACTTCTTTTAAATTTCAAATTGCTCTTAACATTG -873 -872 CGCGAAGAAAATATTAGTTTTATATAATTACCAAAATAAAAAAAAATAAAAATTCAATCAACAAAATAATCTATAAATA -793 -792 GATTTAGAATGTTGCATGATATATATATATATATTTAAAAAACCATGCATGTGTCCTAATCTTGTGAAGTTTTTTTTTTC -713 -712 CATTGAAAATACCAAAAATAACCATTTGTTTATATTTTAATATTAGTTCCATGGCATACCGCCCTGGAAAAGGAAAAATT -636 -632 CATGTAAATAATATATTTTATAAAAAATTTATATTAAAACTAAAATAAAATTTTAGTTGAAATAGTTAAGTTAAAAAGAGTA -553 -552 AAATTATGAGTCATAAGTTTTAAATCTTATTATAGTTTTATTTATAATAAAAATAAAAAATACATTTATATTTAATATTT - 473 -472 GTTATATGAATATTTTTAACAAATTTTCAATTAAATAGATGCTTGGGTGAATCGTAATACCAATTCAATTAAAGACTTAA -393 -392 TATATAAAAGTATATATACAAACAATTATAAATCAATTACTTTTAATAAAATGGATCATTCTGACAATAAATGCAGTT -313 -312 ATAAATACATCAAATGTAAATCTCATGTTTATAAGAAAACACCTAGAAAAAAGTTAAACCAATATTTGAGTCCTAGCTGT -233 -232 GGAGGCATGATTGAGTGAAATCAAATGGACGCTGGTTTTAATTGTATTGAAAGAAAACAATAATCACGTAGGTTGGCAGT -153 -152 TGAACTAATTGAATGGTCTCAACTTTTAATGTGGTGTGTTAATGTTTGGATCGGATAATCTAACTTACCTAATAGCTA -73 -72 GGAAAGTAAAATTCAGACATCACCCGCTACTACTTTTGGCTATAAAAACCCTCCTACCCTCAAGCCCTAACCACGACAAT 8 9 CAGCAATAGTACTACTACTCCAAGCAAGCATTTTCCTTACAAGTTTGTTTTTCTTGTGATTAATCGATATGGTAGCTCA 88 ATG

Fig. 2. Nucleotide sequence of cotton LTP7 promoter. Translation initiation codon (ATG) of LTP7 gene is highlighted with violet colour and transcription initiation site $(+1)$ has been indicated with letter $(A)$ and dark red colour. TATA box is located at (-27) position indicated by green colour while CAAT box is highlighted by dark grey colour. G-box and ABRE are highlighted with yellow and light grey colours, respectively.

in all tissues of cotton under same experimental conditions (Fig. 8). Cotton fibres, bombardment with LTP7 promoter (I kb) construct exhibited strong GUS expression (Fig. 9). The transient GUS analysis of 1.5 kb LTP7 promoter showed much weak GUS expression in fibres. No GUS expression was observed in root, stem and leaf (Fig. 10.) The transient GUS analysis of full length1.8 kb LTP7 promoter showed very strong
GUS expression in fibre tissues. There was no GUS expression in cotton root, stem and leaf (Fig. 11).

Genetic engineering is most powerful tool for introducing genes which are involved in fibre improvement. Cotton fibre is an important food product used universally as raw material for manufacture of fabrics. Molecular breeding approaches are being used to improve quality of fibre for further use in textile industry. 
Table 4. Important regulatory motifs in LTP7 promoter, its $1.8 \mathrm{~kb}, 1.5 \mathrm{~kb}$ and $1 \mathrm{~kb}$ fragment

\begin{tabular}{llll}
\hline \hline Motif name & $1.8 \mathrm{~kb}$ & $1.5 \mathrm{~kb}$ & $1 \mathrm{~kb}$ \\
\hline ARRIAT & 23 & 18 & 14 \\
MYBIAT & 7 & 6 & 5 \\
MYBST1 & 1 & 1 & 1 \\
MYB2AT & 3 & 3 & 1 \\
ABRELATERD1 & 2 & 2 & 1 \\
CCAAT BOX & 5 & 4 & 2 \\
GT1GMSAM4 & 5 & 5 & 3 \\
GATA box & 9 & 6 & 4 \\
WBOXNTERF3 & 1 & 1 & 1 \\
PIF3 & 1 & 0 & 0 \\
DPBFCOREDCD3 & 1 & 0 & 0 \\
MBS & 3 & 2 & 2 \\
TCA element & 2 & 1 & 0 \\
AC1 motif & 1 & 1 & 1 \\
5' UTR pr rich stretch & 1 & 0 & 0 \\
GAREAT & 3 & 2 & 1 \\
SREATMSD & 1 & 1 & 1 \\
TBOXATGAPB & 1 & 1 & 0 \\
SV40COREENHAN & 2 & 2 & 1 \\
ERELEE4 & 1 & 1 & 1 \\
ANAERO1CONSENSUS & 2 & 2 & 1 \\
\hline \hline
\end{tabular}

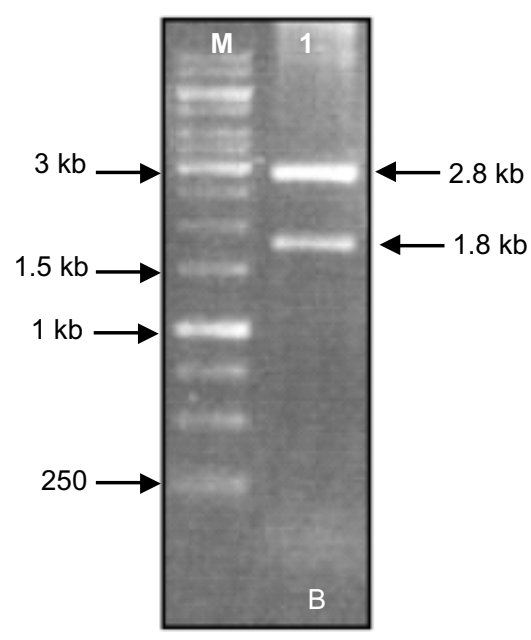

Fig. 3. Restriction digestion of full length LTP7 promoters cloned in TA cloning vector. (A) M: 1 kb DNA ladder; Lane 1 shows restriction digestion of clone using SacI and ApaI releasing $2 \mathrm{~kb}$ SPS promoter along with $2.8 \mathrm{~kb}$ vector back bone. (B) M: $1 \mathrm{~kb}$ DNA ladder; Lane 1: Restriction analysis showing $1.8 \mathrm{~kb}$ LTP7 promoter and $2.8 \mathrm{~kb}$ vector back bone.

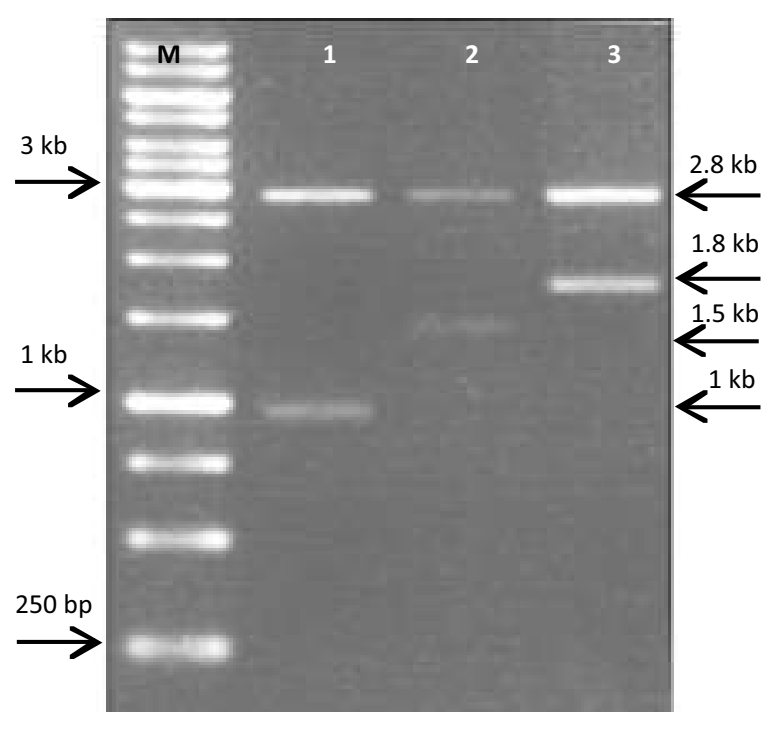

Fig. 4. Cloning of promoter deletion fragments in TA cloning vector. B: Restriction digestion of LTP7 promoter and its fragment with SacI and ApaI. M: 1 kb ladder marker; Lanes 1,2 and 3 represent $1 \mathrm{~kb}, 1.5 \mathrm{~kb}$ and $1.8 \mathrm{~kb}$ LTP7 promoter fragments, respectively along with $2.8 \mathrm{~kb}$ vector back bone.

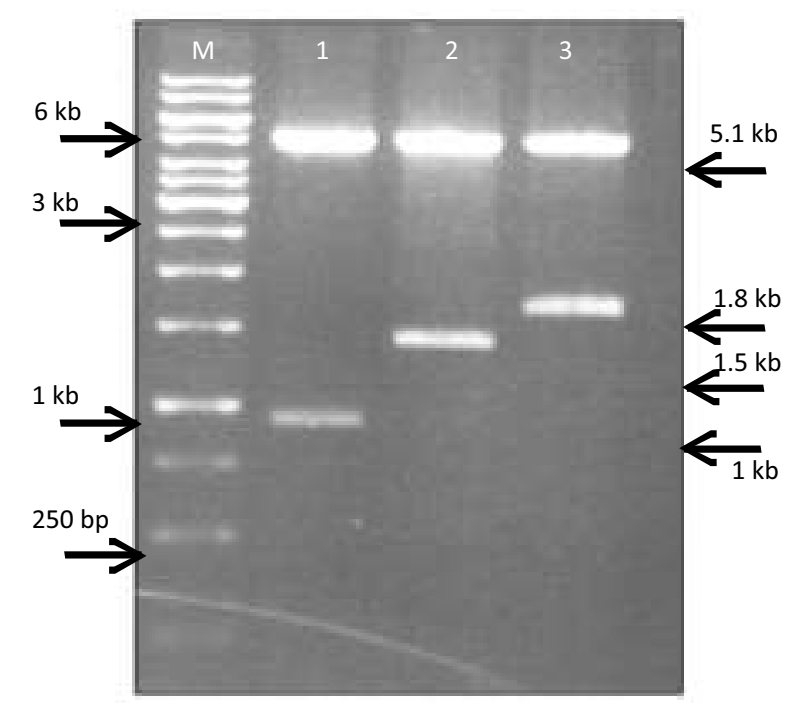

Fig. 5. Cloning of LTP7 promoter and its deletion fragments in Plant expression vector pGR1. Restriction digestion of promoter fragments cloned in pGR1 with Sac1/HindIII to confirm clones. M: $1 \mathrm{~kb}$ DNA ladder, Lanes 1, 2 and 3 represent $1 \mathrm{~kb}, 1.5 \mathrm{~kb}$ and I.8 kb LTP7 promoter fragments, respectively along with $5.1 \mathrm{~kb}$ vector backbone. 

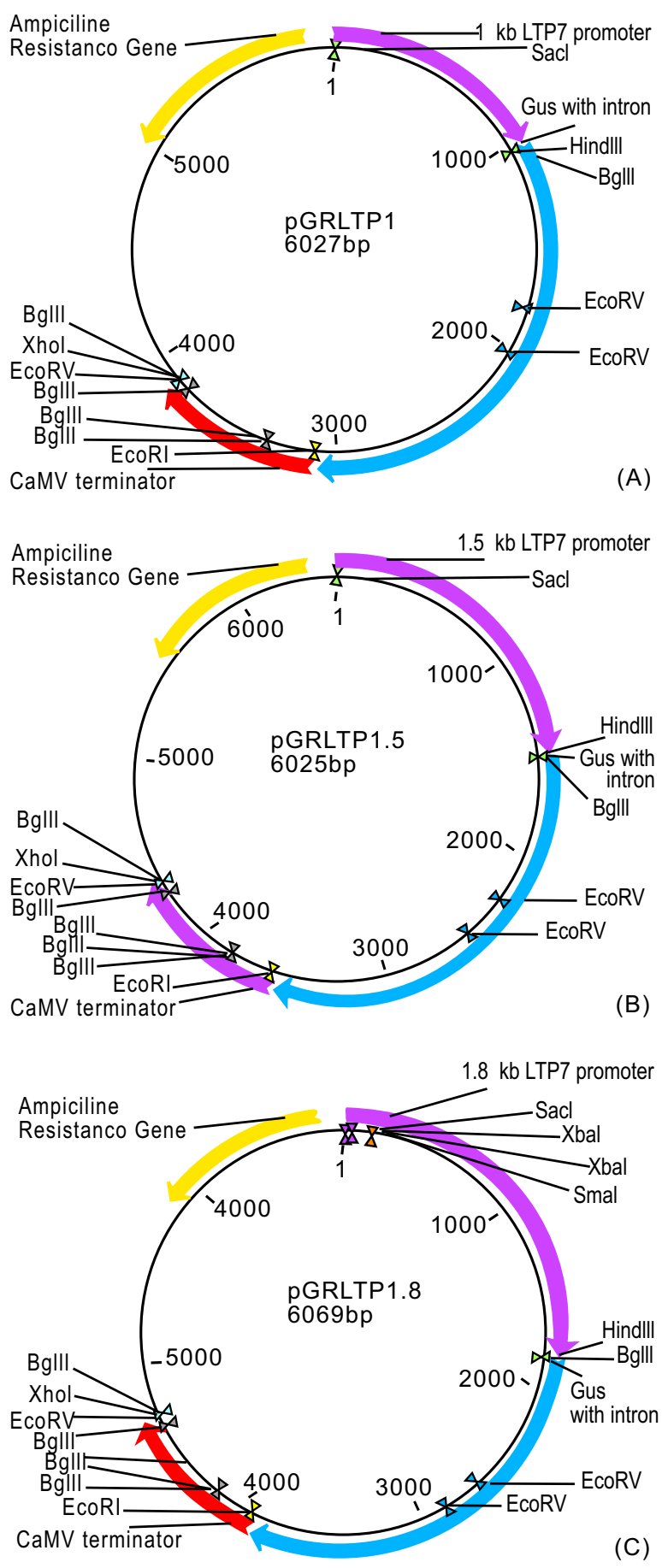

Fig. 6. Vector maps of plasmid constructs containing LTP7 promoter and deletion fragments cloned in pGR1. A: Physical map pGRLTP1 (expression cassette having $1 \mathrm{~kb}$ LTP7 promoter). B: Physical map of pGRLTP1.5 (expression cassette having 1.5 kb LTP7 promoter). C: Physical map of pGRLTP1.8 (expression cassette containing $1.8 \mathrm{~kb}$ LTP7 promoter).
The focus of present research was to isolate and evaluate novel upstream regulatory sequences of fibre related genes. HTGS sequence of LTP7 was analyzed using bioinformatics based analysis tools approaches. Patent BLAST revealed that nucleotide sequence of LTP7 promoter (Accession no. 202820) had 32\% query coverage with previously reported sequence GP235721 (Table 1) means these novel sites can be analyzed and evaluated as promoter regions.

Analysis of promoter sequence through plantCARE identified various $c i s$-acting motifs in LTP7 promoters (Table 2). In LTP7 promoter TATA box was located at position of $-27 \mathrm{bp}$ from transcription initiation site (Fig. 1). Likewise CAAT box motif that is having role in enhancing transcriptional activity (Dai et al., 1999) of promoter was also located at in LTP7 promoters. Endosperm specific elements names as Skn-1 motif
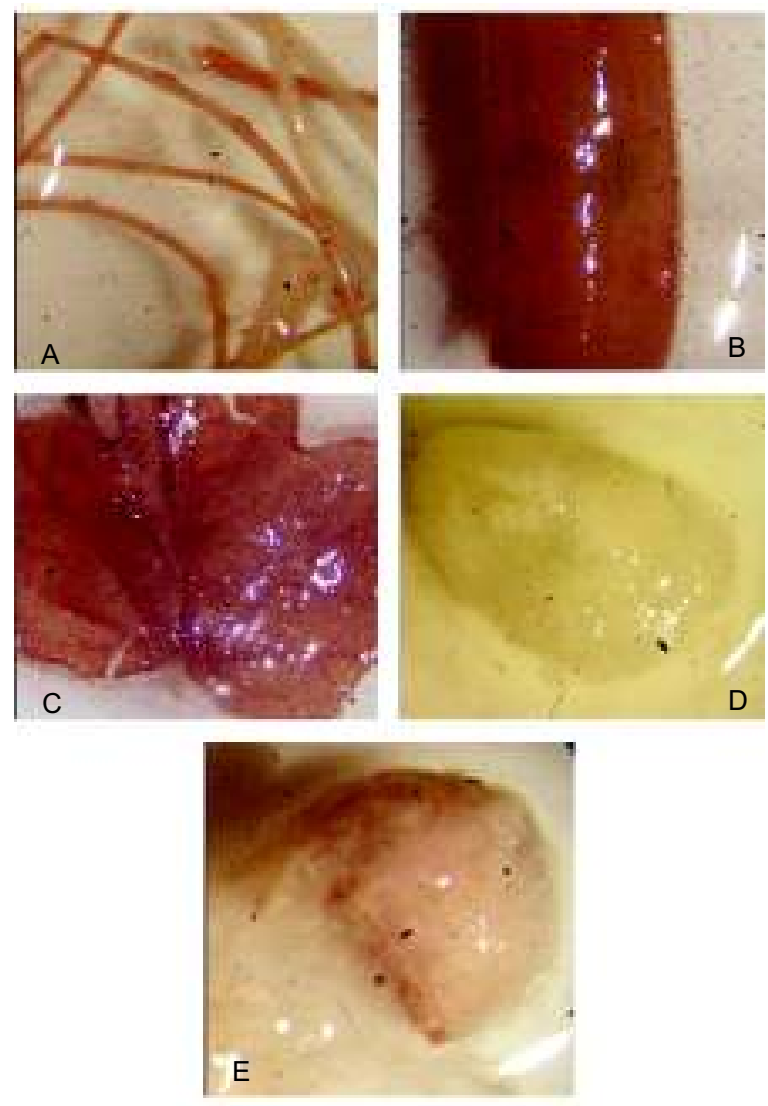

Fig. 7. Transient GUS expression of promoterless construct after bombardment on cotton tissues. (A) root, (B) stem, (C) leaf, (D) 5 DPA fibres and (E) 15 DPA fibres. 

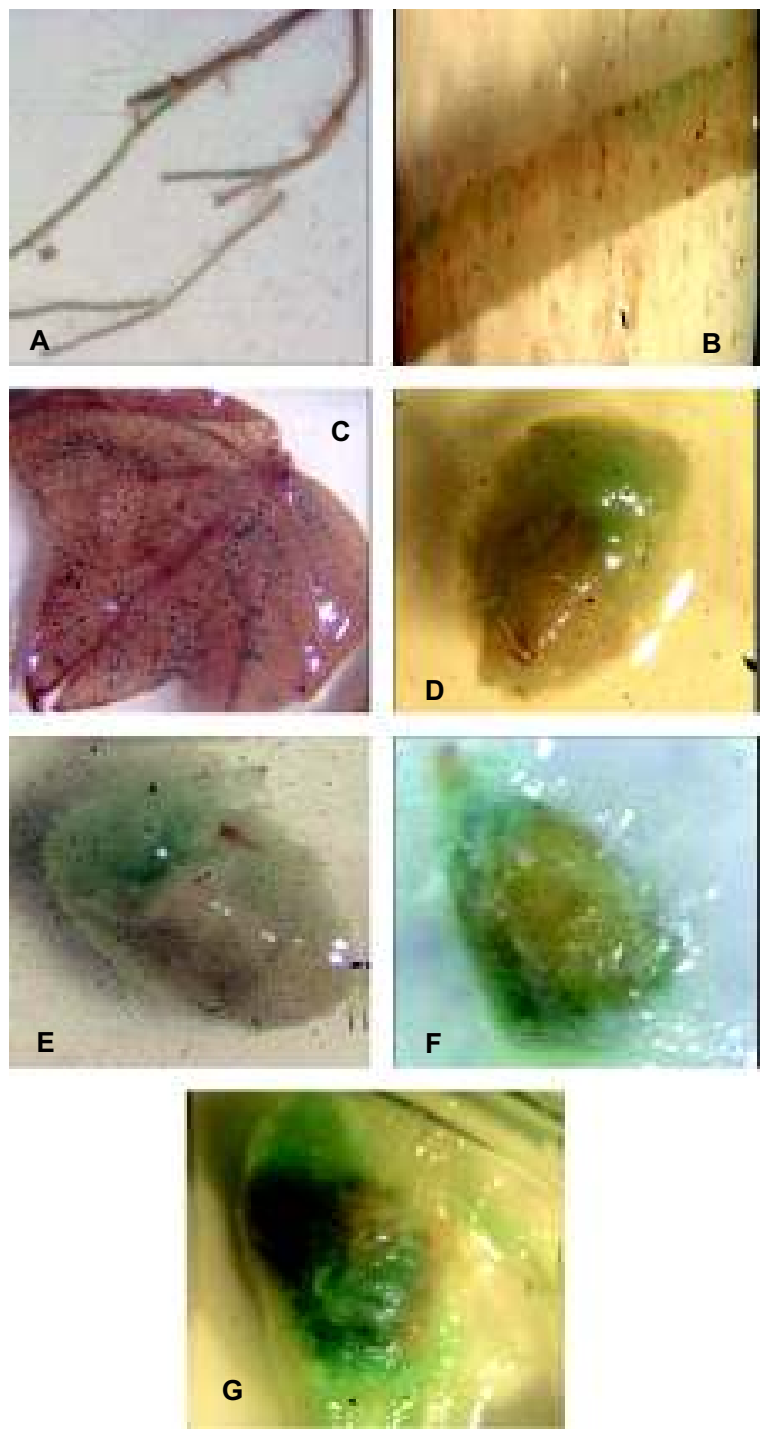

Fig. 8. Transient GUS expression of $35 \mathrm{~S}$ promoter. Transient GUS expression in cotton tissues bombarded with $35 \mathrm{~S}$ promoter. (A) root, (B) stem, (C) leaf, (D) 5 DPA fibres, (E) 10 DPA fibres, (F) 15 DPA fibres and (G) 20 DPA fibres.

(GTCAT) and GCN4 motif (TGTGTCA) were also present in LTP7 promoter. Another motif 5' UTR pyrich stretch that shows high gene expression (Daraselia et al., 1996) was also identified in LTP7 promoter. However, an additional seed specific motif named RY element (CATGCATG) was found in LTP7 promoter as described by Yoshino et al. (2006). LTP7 promoters also contained two MYB sites that bind specifically with the MYB transcription factors. MYB are the largest
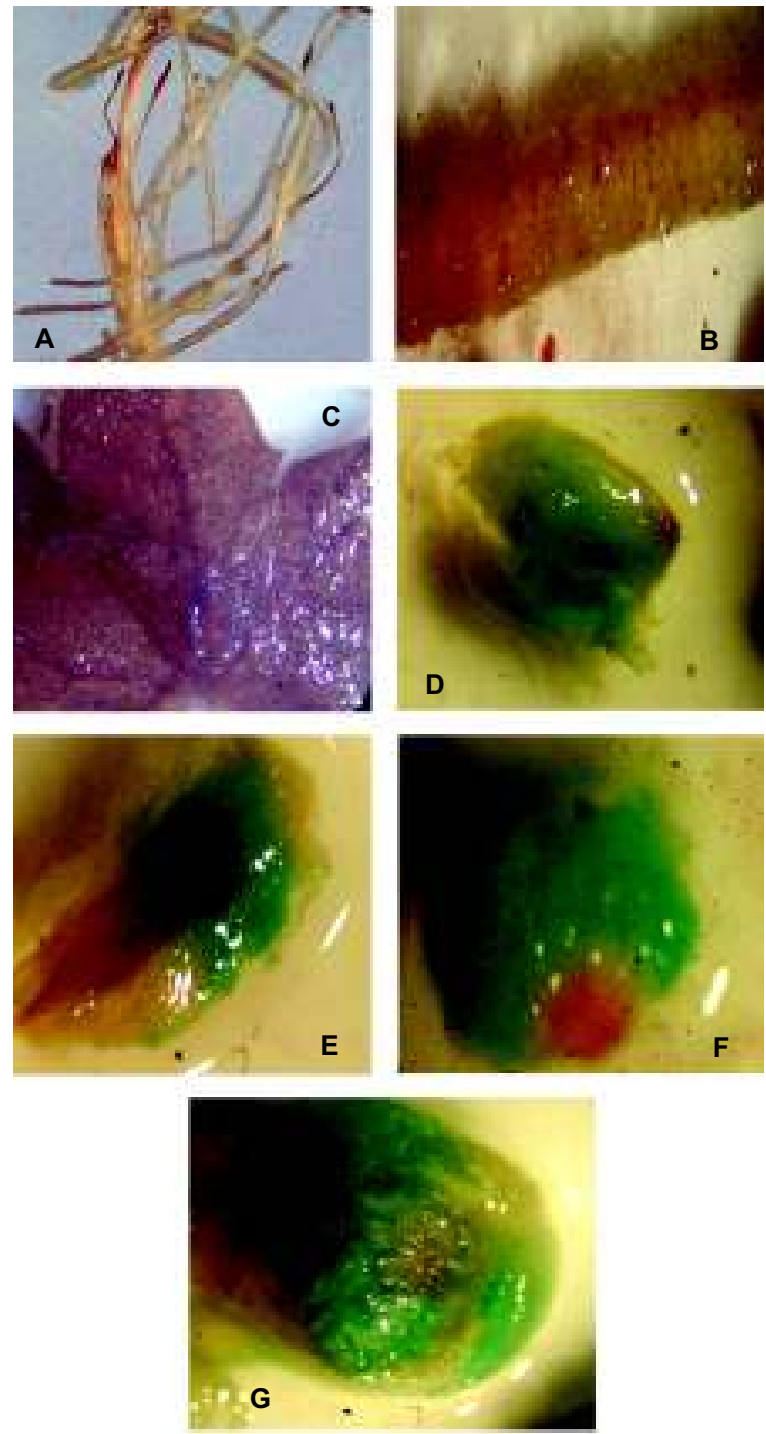

Fig. 9. Transient GUS expression of $1 \mathrm{~kb}$ LTP7 promoter. Transient GUS expression of 1 kb LTP7 promoter in different cotton tissues. (A) root, (B) stem, (C) leaf, (D) 5 DPA fibres, (E) 10 DPA fibres, (F) 15 DPA fibres and (G) 20 DPA fibres.

transcription factors family having diverse functions in plants like response to various biotic and abiotic stresses (Yang et al., 2012; ; Kaur et al., 2010; Shangan et al., 2008). In LTP7 promoter several other MYB binding motifs were also identified that shows good expression in dehydration (Abid et al., 2009). In LTP7 promoter TC rich repeat was also found that shows responsive to stress or fungus attack in previously reported promoters (Daiz-De-Leon et al., 1993). A number of other 

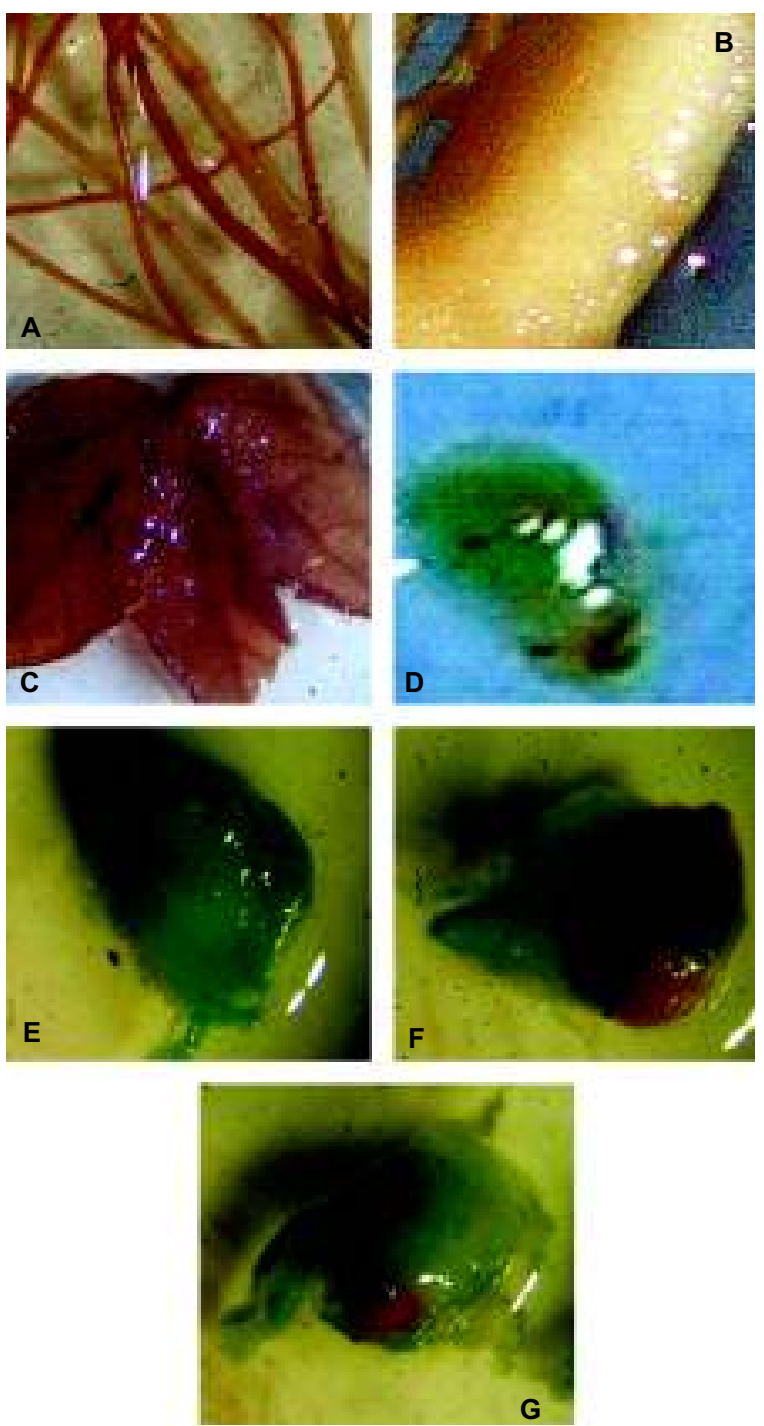

Fig. 10. Transient GUS analysis of $1.5 \mathrm{~kb}$ LTP7 promoter. Expression of GUS gene under control of $1.5 \mathrm{~kb}$ LTP7 promoter in different cotton tissues. (A) root, (B) stem, (C) leaf, (D) 5 DPA fibres, (E) 10 DPA fibres, (F) 15 DPA fibres and (G) 20 DPA fibres.

motifs regulated by light including AE-box, box-I, GT1 motif, G-box and Box-4 were also present in LTP7 promoter (Zhou et al., 2006). G-box is protein binding sequence found in the Rubisco promoter induced by various environmental stimulus (Mary et al., 1992). LTP7 promoter contained additional SORLREP3AT and TBOXATGAPB regions involved in light regulation. A heat shock element with consensus sequence (AAAAAATTTC) was also found in LTP7 that was
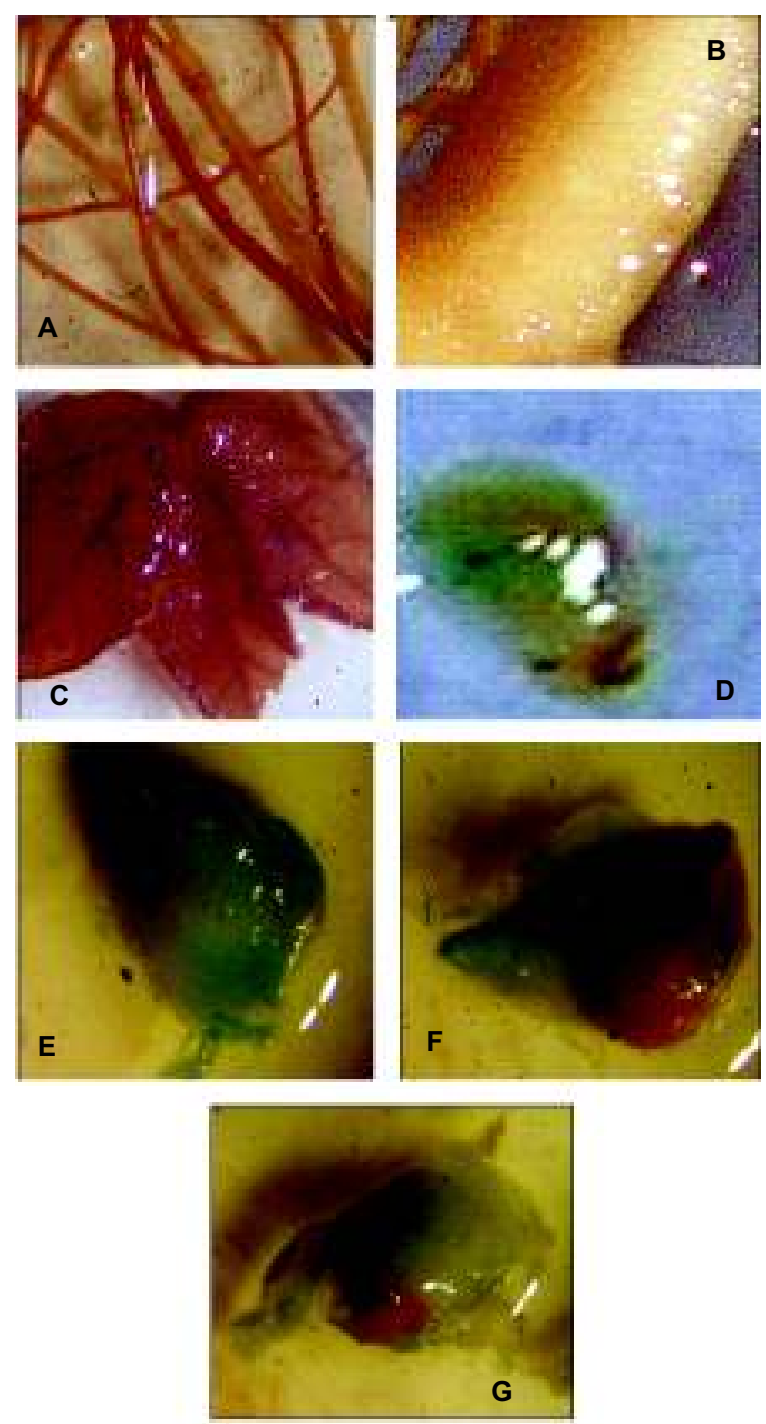

Fig. 11. GUS expression analysis of $1.8 \mathrm{~kb} \mathrm{LTP7}$ promoter. Transient GUS expression of $1.8 \mathrm{~kb} \mathrm{LTP7}$ promoter in cotton tissues. (A) root, (B) stem, (C) leaf, (D) 5 DPA fibres, (E) 10 DPA fibres, (F) 15 DPA fibres and $(G) 20$ DPA fibers.

reported to have role in heat stress responsiveness (Nover et al., 2001). Several number of motifs related to regulation of transcription were present in LTP7 promoter as reported in tomato ataB gene promoter (Sakai et al., 2000). SREATMDS and SURE1STPAT21 motifs were also identified in LTP7 that were previously found in cotton glucuronosyl transferase gene (GhGlcAT1) promoter (Wu et al., 2009). Similarly LTP7 motifs including root hair specific motif 
RHERPATEXPA7, embryo specific motifs named MYBCORE and DPBFCOREDCDC3 while AP1 was floral organ specific motif. Sequence comparison of LTP7 with previously reported promoters LTP3 and FsLTP4 revealed several common regulatory motif in these promoters like G-BOX, HSE, MRE, BOX-4, BOX-I, and MBS.

For expression studies, promoter fragments were cloned into expression vector $\mathrm{pGR} 1$ by replacing $35 \mathrm{~S}$ promoter upstream of GUS gene followed by CaMV terminator. Biolistic transformation is a useful approach to study tissue specific expression of cotton promoters (Kim et al., 2003). GUS expression pattern after biolistic transformation depicted that LTP7 promoter and its deletion fragments revealed that full length promoter and its $1 \mathrm{~kb}$ deletion fragment exhibited strong expression in fibre tissues (Fig. 9-11). However, $1.5 \mathrm{~kb}$ deletion fragment showed weak GUS expression (Fig. 10). In non-fibre tissues, no GUS activity was exhibited by 1.8 $\mathrm{kb}$ promoter and its both deletion fragments. Deletion of $500 \mathrm{bp}$ resulted in lowering expression in fibre tissues. In full length $1.8 \mathrm{~kb}$ single $5^{\prime}$ UTR py-rich stretch was present which was deleted in its deletion mutants. Other motif related to phytochrome regulated expression was deleted in $1.5 \mathrm{~kb}$ fragment. A number of transcription binding sites related to high transcription including GATA box and MYBST1 were trimmed in $1.5 \mathrm{~kb}$ fragment. There may be some negative regulatory region $-500 \mathrm{bp}$ away from TiS. This showed that regulatory transcription elements present away from core promoter region also play important role in modulating gene expression as observed $1.5 \mathrm{~kb}$ deletion mutant. Surprisingly, strong expression in fibre again appeared in $1 \mathrm{~kb}$ fragment. In $1 \mathrm{~kb}$ LTP7 promoter although 5'UTR py-rich stretch was deleted but 4 GATA motifs along with SV40COREENHAN and single MYB binding sites were present in this region. Cotton tissues were bombarded with $35 \mathrm{~S}$ promoter (positive control) and promoter less construct (negative control) to avoid false results. Regeneration in cotton is a time consuming process. Therefore transient GUS assay was used to overcome problems with somatic embryogenesis and regeneration in cotton. This has proved very useful protocol to detect promoter activity in fibres of different developmental ages. Newly isolated LTP7 promoter have wide potential applications for use to express genes in fibre tissues of cotton especially during elongation period that is the most crucial stage of fibre development. Elongation phase determines the length of cotton fibre that is most desirable for textile industry. Therefore, this promoter may be used to express fibre genes specifically during elongation phase in fibres.

Conflict of Interest. The authors declare no conflict of interest.

\section{References}

Abid, G., Silue, S., Muhovski, Y., Jacquemin, J.M., Toussaint, A., Baudoin, J.P. 2009. Role of myoinositol phosphate synthase and sucrose synthase genes in plant seed development. Gene, 439: 1-10.

Allen, D., Trevor, H.Y., Jocelyn, K.C.R., David, B., Reinhard, J., Ljerka, K., Lacey, K., Lacey, S. 2009. Arabidopsis LTPG is a glycosyl phosphatidyl inositol-anchored lipid transfer protein required for export of lipids to the plant surface. Plant Cell, 21: 1230-1238.

Dai, Z., An, K., Edward, G.E., An, G. 1999. Functional role of CAAT Box element of the opaline synthase (nos) promoter. Journal of Plant Biology, 42: 181185.

Daiz-De-leon, F., Klotz, K.L., Legrimini, L.M. 1993. Nucleotide sequence of the tobacco (Nicotiana tabacum) onion peroxide gene. Plant Physiology, 101: 1117-1118.

Daraselia, N.D., Tarchevskaya, S., Notita. T.O. 1996. The promoter of tomato 3-hydroxyl-3-methyl glutaryl co-enzyme reductase gene 2 has unusual regulatory elements that direct high-level of expression. Plant Physiology, 112: 727-733.

Fleming, A.J.T., Mandel, S., Hofmann, P., Sterk, S.C., Vries, D., Kuhlemeier, C. 1992. Expression pattern of a tobacco lipid transfer protein gene within the shoot apex. Plant Journal, 2: 855-862.

Gomes, E., Sagot, C., Gaillard, L., Laquitaine, B., Poinssot, B., Sanejouand. Y.H., Delrot, S., CoutosThe'venot, P. 2003. Non specific lipid transfer protein genes expression in grape (Vitis sp.) cells in response to fungal elicitor treatments. Molecular PlantMicrobe Interaction, 16: 456-464.

Kaur, H., Herinzel, N., Schöttner, M., Baldwin, I.T., Ivan, G. 2010. R2R3-NaMYB8 regulates the accumulation of phenylpropanoid polyamine conjugates which are essential for local and systemic defense against insect herbivores in Nicotiana attenuate. Plant Physiology, 152: 1731-1747.

Kim, K.Y., Kwon, S.Y., Lee, H.S., Hur, Y., Bang, J.W., Kwak, S.S. 2003. A novel oxidative stress-inducible 
peroxidase promoter from sweet potato: molecular cloning and characterization in transgenic tobacco plants and cultured cells. Plant Molecular Biology, 51: 831-838.

Liu, H.C., Creech, R.G., Jenkins, J.N., Ma, D.P. 2000. Cloning and promoter analysis of the cotton lipid transfer protein gene Ltp3. Biochimica Biophysica Acta, 1487: 106-111.

Machando, A., Wu, Y., Yang, Y., Llewellyn, D.J., Dennis, E.S. 2009. The MYB transcription factor GhMYB25 regulates early fibre and trichome development. Plant Journal, 59: 52-62.

Mary, E., Foster, R., Chua, N.H. 1992. Sequences flanking the hexameric G-box core CACAGTG affect the specificity of protein binding. Plant Cell, 4: 485-496.

McGee, T.P., Skinner, H.B., Whitters, E.A., Henry, S.A., Bankaitis, V.A. 1994. A phosphatidyl inositol transfer protein controls the phosphatidyl choline content of Golgi membranes. Journal of Cell Biology, 124: 273-287.

Nover, L., Bharti, K., Döring, P., Mishra, S.K., Anguli, A., Scharf, K.D. 2001. Arabidopsis and the heat shock transcription factors world: how many heat stress transcription factors do we need. Cell Stress Chaperon, 6: 177-189.

Sakai, H., Aoyama, T., Oka, T. 2000. Arabidopsis ARR1 and ARR2 response regulators operate as transcriptional activators. Plant Journal, 24: 703-711.

Shangguan, X.X., Xu, B., Yu, Z.X., Wang, L.J., Chen, X.Y. 2008. Promoter of a cotton fibre MYB gene functional in trichomes of Arabidopsis and glandular trichomes of tobacco. Journal of Experimental Botany, 59: 3533-3542.

Wu, Y.F., Xu, W.L., Huang, G.Q., Gong, S.Y., Li, J. 2009. Expression and localization of GhH6L, a putative classical arabinogalactan protein in cotton (Gossypium hirsutum). Acta Biochimica et Biophysica Sinica, 41: 495-503.

Yang, Y., Costa, A., Leonhardt, N., Siegel, R.S., Schroeder, J.I. 2012. Isolation of a strong Arabidopsis guard cell promoter and its potential as a research tool. Plant Methods, 4: 6.

Sakai, H., Aoyama, T., Oka, T. 2000. Arabidopsis ARR1 and ARR2 response regulators operate as transcriptional activators. Plant Journal, 24: 703711.

Zhao, T.J., Sun, S., Liu, Y., Liu, J.M., Liu, Q., Yan, Y.B., Zhou, H.M. 2006. Regulating the droughtresponsive element (DRE)-mediated signaling pathway by synergic functions of trans-active and transinactive DRE binding factors in Brassica napus. Journal of Biological Chemistry, 281: 1075210759. 